\title{
Superlubricity and tribochemistry of polyhydric alcohols
}

\author{
C. Matta, ${ }^{1}$ L. Joly-Pottuz, ${ }^{2}$ M. I. De Barros Bouchet, ${ }^{1}$ and J. M. Martin ${ }^{1}$ \\ ${ }^{1}$ Ecole Centrale de Lyon, LTDS, 36 Avenue Guy de Collongue, 69134 Ecully, France \\ ${ }^{2}$ INSA de Lyon, MATEIS, 7 Avenue Jean Capelle, 69621 Villeurbanne, France

\begin{abstract}
M. Kano
Nissan Research Center, Kanagawa Industrial Technology Center, 705-1, Shimo-imaizumi, Ebina, Kanagawa 243-0435, Japan
\end{abstract}

Qing Zhang and W. A. Goddard III

Materials and Process Simulation Center, MS 139-74, California Institute of Technology, Pasadena, California 91125, USA

(Received 26 September 2007; revised manuscript received 2 April 2008; published 28 August 2008)

\begin{abstract}
The anomalous low friction of diamondlike carbon coated surfaces lubricated by pure glycerol was observed at $80^{\circ} \mathrm{C}$. Steel surfaces were coated with an ultrahard $1 \mu \mathrm{m}$ thick hydrogen-free tetrahedral coordinated carbon (ta-C) layer produced by physical vapor deposition. In the presence of glycerol, the friction coefficient is below 0.01 at steady state, corresponding to the so-called superlubricity regime (when sliding is then approaching pure rolling). This new mechanism of superlow friction is attributed to easy glide on triboformed $\mathrm{OH}$ terminated surfaces. In addition to the formation of OH-terminated surfaces but at a lower temperature, we show here some evidence, by coupling experimental and computer simulations, that superlow friction of polyhydric alcohols could also be associated with triboinduced degradation of glycerol, producing a nanometer-thick film containing organic acids and water. Second, we show outstanding superlubricity of steel surfaces directly lubricated by a solution of myo-inositol (also called vitamin Bh) in glycerol at ambient temperature $\left(25^{\circ} \mathrm{C}\right)$. For the first time, under boundary lubrication at high contact pressure, friction of steel is below 0.01 in the absence of any long chain polar molecules. The mechanism is still unknown but could be associated with friction-induced dissociation of glycerol and interaction of waterlike species with steel surface.
\end{abstract}

DOI: 10.1103/PhysRevB.78.085436

PACS number(s): 62.20.Qp, 82.40.-g

\section{INTRODUCTION: SUPERLUBRICITY AND LUBRICATION BY GLYCEROL}

Among all physical phenomena, friction perhaps poses one of the greatest challenges to the scientific and industrial communities and is directly linked to energy efficiency. Considering that in most industrialized nations, the annual cost of friction and wear-related energy and material losses is estimated to be $5 \%-7 \%$ of their gross national products, the further reduction (or even elimination) of friction in all kinds of moving mechanical systems would be extremely beneficial to the economical well being of all nations.

The term "superlubricity" was first used by Motohisa Hirano and Kazumasa Shinjo in 1990 to describe a theoretical sliding regime in which friction or resistance to sliding completely vanishes. ${ }^{1}$ Historically, the earliest studies on superlubricity started in the mid-1980s, but the real progress occurred during the 1990s. In particular, theoretical studies by Sokoloff ${ }^{2}$ and Hirano predicted the existence of such superlubricious states between weakly interacting, atomically smooth surfaces. Other researchers also confirmed that such a state of near-zero friction may indeed exist between sliding surfaces of a number of solid materials that are brought into intimate contact in an incommensurate or ideally misaligned/ misfit fashion. By bringing such surfaces from a commensurate to an incommensurate state, these researchers were able to observe a clear transition from a pure stick slip (indicative of high friction) to smooth sliding (indicative of no friction). It should be noted that superlubricity is theoretically applicable to vanishing of static friction but kinetic friction, which is velocity dependent, can survive. Unfortunately, we are presently unable to measure friction coefficients at values below $10^{-3}$. More details can be found in a recently published book on superlubricity. ${ }^{3}$

Very low friction coefficients under moderate contact pressures (megapascal range) in tribological systems are well explained by hydrodynamic and elastohydrodynamic (EHD) lubrication regimes. It is the case for journal bearings with oil and skiing or skating with water, for example. When pressure is a few thousand times higher, such hydrodynamic films cannot formed and friction is typically in the 0.1 range. We briefly explain how the friction coefficient can be calculated in these conditions. The traditional Amontons friction law $F=\mu \mathrm{W}$ relates the friction force $F$ to the normal load via the friction coefficient $\mu$. The Bowden and Tabor ${ }^{4}$ model of friction provides a good starting point for understanding how a thin interface film can drastically reduce the friction coefficient. The friction coefficient is assumed to depend on the normal load $W$, the real contact area $A$, and the shear strength $S$ of the interfacial tribofilm (or film transfer) as

$$
\mu=S \cdot \frac{A}{W} .
$$

The shear strength $S$ of solids at high pressure is generally observed to be pressure dependent, which can be approximated by ${ }^{5}$

$$
S=S_{0}+\alpha P .
$$

According to the Hertzian contact theory (below the elastic limits), and in the sphere-on-plane configuration, the friction coefficient $\mu$ depends of three variables as ${ }^{6}$ 


$$
\mu=S_{0} \pi\left(3^{R} / 4^{E}\right)^{2 / 3} W^{-1 / 3}+\alpha,
$$

where $E$ is the composite elastic modulus of the contacting materials and $R$ is the radius of the sphere. This simplified model assumes that the real contact area corresponds to the Hertzian zone, as calculated in Eq. (3). Therefore, friction measurements at different normal loads can be useful to determine $S_{0}$ and $\alpha$ values in a given tribological system. However, Eq. (3) indicates that the minimum friction coefficient value is $\alpha$ so that the friction cannot vanish completely. For example, friction experiments performed on $\mathrm{MoS}_{2}$ coatings under different atmospheres ${ }^{7-11}$ have led to $\alpha=0.001$. On the other hand, if the shear strength of the interface is very low, say $25 \mathrm{MPa}, \mu$ is predicted to be a few thousandths ( $\mu$ $<0.01$ ), making the measurement of friction very difficult. The accuracy of mechanical devices measuring the friction tangential force and the normal load simultaneously makes it difficult to quantify the friction for $\mu<0.01$, which we refer to as extremely low friction or superlubricity.

In the case of very thin interface films such as H-terminated surfaces on carbon materials, or very thin tribofilms (thickness below $2 \mathrm{~nm}$ ), it is not certain that Eq. (2) and (3) are still valid and the existence of a limiting value to the lowest friction attainable remains questionable. Superlubricious situations were predicted and observed only in ultrahigh vacuum (or inert gas) since 1993 by different authors. . $^{71-13}$

Concerning lubrication by polyhydric alcohols and particularly glycerol $\mathrm{C}_{3} \mathrm{H}_{8} \mathrm{O}_{3}$, selective transfer was the name given in the 1970s to various phenomena involving copperbased materials and ferrous surfaces as friction pairs. It was widely reported to produce extremely low friction in the technical literature of the former USSR. Basically, the mechanism was attributed to the formation of a beneficial metallic film, possibly copper, which has an ability to reduce friction. Later, in the 1990s, the role of colloidal and gel-like products, which result from the tribochemical reaction, was emphasized and the rheology of the colloidal film was thought to play the key role in the friction reduction. At this point, interest of Western tribologists in these ideas was negligible mainly due to the ambiguous definition of the selective transfer mechanism and also low access to Russian literature. However, a recent article on this matter was published in 2006 by Ilie. ${ }^{14}$

Superlubricity experiments in ultrahigh vacuum have already been reported in the literature by some of us, first in 1993 using stoichiometric $\mathrm{MoS}_{2}$ coatings, ${ }^{7}$ in 1996 using hydrogenated carbon films $a$-C: $\mathrm{H}^{11}$ and recently in 2007 using ta-C carbon films and glycerol. ${ }^{15}$ However, superlubricity has never yet been approached using lubrication of steel surfaces in practical situations. Friction coefficients below 0.05 under boundary lubrication of steel samples have never been reported in the literature.

Here, we are most interested in the use of polyhydric alcohols (or polyols) to lubricate different material combinations used in practical situations including silicon and steel. Figure 1 shows chemical formula of well known polyols and particularly cyclic hexols, (cyclitols) such as phenol, resorcinol, pentaerythritol, and myo-inositol. In the past, these poly-

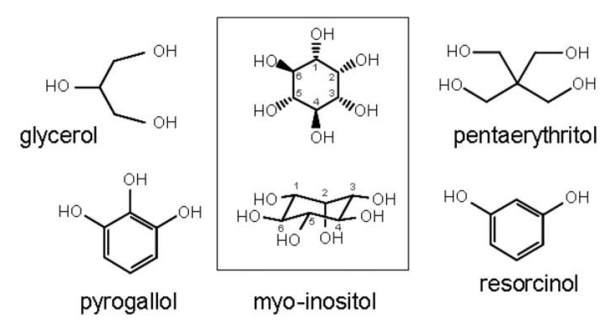

FIG. 1. . Chemical formula of some polyhydric alcohols: glycerol, pyrogallol, resorcinol, pentaerythritol, and myo-inositol (also called vitamin Bh). All these molecules are small and have different shapes such as spherical, crab shape or spider shape. They do not contain aliphatic chains like most of amphiphilic molecules used in traditional lubricants. In this study, we focus on glycerol and myo-inositol.

ols have been widely used to synthesize the corresponding esters as lubricant base oils. However, no attempt has been made to evaluate the intrinsic tribological properties of such polyhydric alcohols. In this work, we will focus on glycerol and myo-inositol that is classified as a member of the vitamin $\mathrm{B}$ complex (often referred to as vitamin $\mathrm{Bh}$, as in the vitamins and health supplements guide) although it can be synthesized by the human body. The chemical formula of myoinositol is $\mathrm{C}_{6} \mathrm{H}_{12} \mathrm{O}_{6}$. In its most stable geometry, the inositol ring is in the chair conformation. There are nine stereoisomers, all of which may be referred to as inositol. However, the natural isomer has a structure in which the first, third, fourth, fifth, and sixth hydroxyls are equatorial, while the second hydroxyl group is axial. All these polyol molecules (including glycerol) are relatively small and have different shapes such as spherical, crab shape or spider shape, etc. They do not contain aliphatic chains like most of amphiphilic molecules used in traditional lubricants. In this paper, we show and discuss results obtained with glycerol on steel, diamondlike coatings (DLCs) and silicon surfaces, and myoinositol directly on steel.

\section{EXPERIMENTAL PART}

The sliding tests were performed using a reciprocating cylinder-on-disk tribometer (SRV) and the lubricant was supplied on the disk before the test by putting a few droplets $(5$ cc) of the test oil heated at $80{ }^{\circ} \mathrm{C}$. The cylinders $(18 \mathrm{~mm}$ diameter and $22 \mathrm{~mm}$ long) were made of AISI 52100 steel and were polished to a surface roughness of Ra equal to $0.05 \mu$. The disk has a diameter of $24 \mathrm{~mm}$ and is $7.9 \mathrm{~mm}$ thick, and it is made of carburized steel. The cylinder was loaded on the stationary disk by a force of $400 \mathrm{~N}$ that generated a maximum contact pressure of $270 \mathrm{MPa}$. The width of the wear track was $3.0 \mathrm{~mm}$ at $50 \mathrm{~Hz}$ reciprocating movement and the duration of the test was $15 \mathrm{~min}$. In these conditions, the sliding speed is $0.3 \mathrm{~m} \mathrm{~s}^{-1}$. In these testing conditions and assuming a ta-C coating surface roughness of about $30 \mathrm{~nm}$, a mixed EHD and boundary lubrication regime was achieved, indicating that any lubricant film inside the contact has a nanometer scale thickness.

The gas phase lubricated friction experiments and the UHV friction tests were carried out with a reciprocating pin- 
on-flat tribometer located in an UHV chamber equipped with a gas analyzer to follow partial pressure of introduced gases during the friction test. This new apparatus called environmentally controlled tribometer (ECT) displays several advantages. First, it allows to monitor the introduced gas pressure from $10^{-9}$ to $10^{3} \mathrm{hPa}$ and in addition to pure gas, gases mixtures can also be introduced. Second, the temperature of the flat specimen can be controlled from liquid nitrogen temperature to $600{ }^{\circ} \mathrm{C}$ (see details on this tribometer in ${ }^{16}$ ). The stationary hemispherical pin was loaded against the flat at $80{ }^{\circ} \mathrm{C}$ by a force of $1 \mathrm{~N}$ that generated a contact pressure of $345 \mathrm{MPa}$ and the sliding speed was $0.001 \mathrm{~m} \mathrm{~s}^{-1}$. Only boundary lubrication is present in these conditions.

The reciprocating pin-on-flat sliding tests conducted in the liquid phase were also used. The hemispherical pin, 10 $\mathrm{mm}$ long, presents a radius of curvature of $8 \mathrm{~mm}$ and was made of hardened bearing steel (AISI 52100) and polished to a surface roughness of $\mathrm{Ra} 0.05$ micron. The flat measured $10 \times 8 \times 2 \mathrm{~mm}^{3}$ was also made of AISI 52100 steel. The contact pressure at the sliding interfaces was varied from 330 to $830 \mathrm{MPa}$. The sliding speed was constant at $0.003 \mathrm{~m} \mathrm{~s}^{-1}$. Lubrication was provided by an oil bath at room temperature $\left(23{ }^{\circ} \mathrm{C}\right)$.

The ta-C DLC coating was applied to the polished carburized steel disk and the hardened steel pins to a thickness of $0.5 \mu$ from a graphite target by arc-ion plating, a physical vapor deposition (PVD) process, and did not contain hydrogen. The hydrogen-containing DLC $(a-\mathrm{C}: \mathrm{H})$ coating was applied to the polished carburized steel disk and hardened steel pins to a $1.0 \mu$ thickness by a plasma-assisted chemical vapor deposition process from hydrocarbon gas and contained about 20 at. \% of hydrogen. We evaluated the friction properties of two DLC couples (ta-C and $a-\mathrm{C}: \mathrm{H}$ ) lubricated with glycerol at $80{ }^{\circ} \mathrm{C}$ and under ultrahigh vacuum (UHV) in order to clarify the origin for superlow friction. The ta-C coating was deposited by PVD using cathodic arc plating. ${ }^{15}$ The percentage of $s p^{3}$ sites in the ta-C coating is $90 \%$ as calculated from $\mathrm{x}$-ray appearance near-edge structure experiments. ${ }^{17}$ This result is consistent with the high values measured by nanoindentation for hardness $(\sim 65 \mathrm{GPa})$ and Young modulus $(650 \mathrm{GPa}) .{ }^{17}$ In addition, secondary ion mass spectroscopy (SIMS) profiling experiments detected no hydrogen in the bulk of the ta-C coating. ${ }^{3,17}$ Thus, the carbon coating mostly corresponds to "amorphouslike diamond" or tetrahedral amorphous carbon (or ta-C), in the carbonhydrogen phase diagram terminology of Robertson. ${ }^{18}$

The glycerol tested in this study is a commercial product from Carlo Erba. Its purity is estimated to be $99.5 \%$ and its density is 1.257 at $25{ }^{\circ} \mathrm{C}$. The commercial myo-inositol is provided in the form of white powder by Acros Organics Co. Its purity is estimated to be more than $98 \%$. To carry out the tribological tests, it is diluted in glycerol with a concentration of $1 \%$ in mass. Deuterated glycerol was a commercial product from Isotec Co.

Because the situation is far beyond the limits of most of classical surface analysis techniques, we used molecular dynamics (MD) simulations to study the friction of ta-C surfaces in the presence of glycerol molecules. The molecular dynamics studies used the ReaxFF reactive force field based on quantum mechanics calculations of structures and reac- tions of many carbon-based systems. ${ }^{19,20}$ Previous studies have documented the accuracy of ReaxFF to describe reactions and to prepare structures for amorphous systems. In order to obtain an amorphous carbon structure from simulations, we started with a periodic cell containing 512 carbon atoms with the diamond structure $(4 \times 4 \times 4$ superlattice $)$, heated this system to $8000 \mathrm{~K}$ for 2 ps to form the liquid phase, then quenched the system to $300 \mathrm{~K}$ at a cooling rate of 1400 and $100 \mathrm{~K} / \mathrm{ps}$, and finally equilibrated the structure at $300 \mathrm{~K}$ for $3 \mathrm{ps}$. The process was carried out for densities ranging from 2.7 to $3.4 \mathrm{~g} / \mathrm{cm}^{3}$. The ta-C surface models synthesized with simulation match well with our experimental characterizations of these surfaces. For ta-C of $3.24 \mathrm{~g} / \mathrm{cm}^{3}$, there are $71.5 \% s p^{3}$ carbon atoms, $28.1 \% s p^{2}$ and $0.4 \% s p^{1}$ in the simulation model, which are consistent with our x-ray-excited auger electron spectroscopy (XAES) experimental results, $70 \% s p^{3}$ carbon at the same density. The ta-C model with $3.18 \mathrm{~g} / \mathrm{cm}^{3}$ density was used in the sliding simulation.

To construct the surface of ta-C, we cut through the cell with planes perpendicular to each of the $x, y$, and $z$ directions. For each direction, we attempted to cut the solid at 30 places, equally spaced at $0.5 \AA$ and spanning the periodic supercell of $14.75 \AA$. In each case, the two parts of the ta-C were first separated by $1.5 \AA$ and the positions of the atoms were optimized to minimize the energy. Then, we selected the best plane from each of the three sets and carried out MD simulation at $300 \mathrm{~K}$ while separating the planes by a total of $2.5 \AA$. Then, we selected the best of these cases as the actual surface of this particular ta-C structure. By using this surface, we carried out sliding simulations by moving the top surface with respect to the bottom one.

The ta-C/ta-C sliding couple was constructed by bringing two ta-C slabs into contact. Periodic boundary conditions were imposed in the $x-y$ plane, while about $45-48 \AA$ of vacuum was allowed in the $z$ direction. The bottom $0.9 \AA$ of the lower slab (with 32 atoms/cell) were held rigid in all simulations, while the top $0.9 \AA$ of the upper slab was slid at a constant velocity of $1 \mathrm{~nm} / \mathrm{ps}$ along the sliding direction. At each point, all remaining 480 atoms were allowed to freely move according to the forces. An external force was imposed along the $z$ direction (perpendicular to the slabs) to keep the distance between the centers of mass of the two slabs constant. This provides the normal load. Starting with the initial interface models at an initial temperature of $300 \mathrm{~K}$, we carried out constant energy MD simulations while keeping the cell parameters fixed.

\section{RESULTS AND DISCUSSION}

\section{A. Anomalous low friction of diamondlike carbon in presence of glycerol}

Figure 2 shows summary of steady-state friction data obtained for different material combinations with the different tribometers described, and in three different selected environments at $80{ }^{\circ} \mathrm{C}$ : (i) ultrahigh vacuum $(10 \mathrm{nPa}$ partial pressure), (ii) in presence of liquid pure glycerol, and (iii) in presence of glycerol+1 wt \% of myo-inositol (cis-1,2,3,5trans-4,6-cyclohexanehexol). The superlubricity of $a-\mathrm{C}: \mathrm{H}$ 


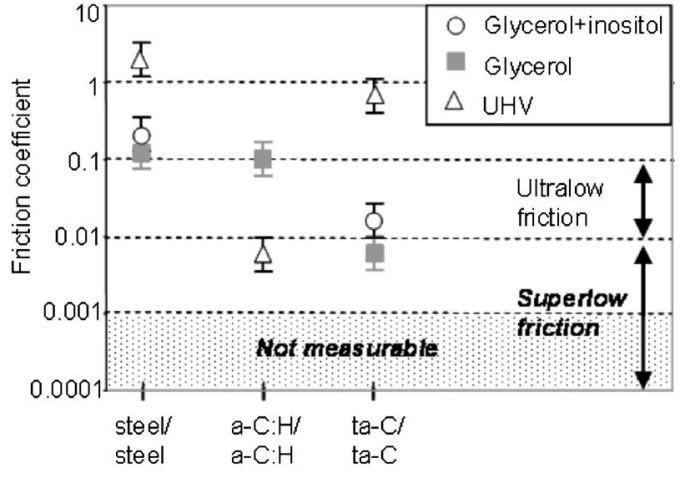

FIG. 2. Steady-state friction coefficients values obtained with steel/steel, $a-\mathrm{C}: \mathrm{H} / \mathrm{a}-\mathrm{C}: \mathrm{H}$, and ta-C/ta-C friction pairs materials, lubricated in three different environments. All tests were performed at a temperature of $80{ }^{\circ} \mathrm{C}$. The friction tests in UHV were performed with the ECT tribometer. The friction tests in liquid pure glycerol and glycerol $+1 \mathrm{wt} \%$ myo-inositol were performed with a SRV machine at $270 \mathrm{MPa}$ contact pressure and $0.3 \mathrm{~m} \mathrm{~s}^{-1}$ sliding speed. Superlubricity is achieved for the first time with ta- $\mathrm{C}$ under boundary lubrication regime with glycerol. In these conditions, sliding is approaching pure rolling.

coatings under ultrahigh vacuum is observed and has previously been reported by several research groups, including some of us. ${ }^{21}$ Although ta-C was little studied in details in the literature, we obtained amazing friction results approaching superlubricity for the ta-C/ta-C combination under boundary lubrication conditions with pure glycerol at $80{ }^{\circ} \mathrm{C}$. As shown in Fig. 2, the friction coefficient of the lubricated ta-C friction pairs is drastically lower than the friction of both $a-\mathrm{C}: \mathrm{H} / \mathrm{a}-\mathrm{C}: \mathrm{H}$ and steel/steel combinations. These results strongly suggest that the superlow friction behavior involves the interaction between the ta-C coating material and the alcohol groups contained in the glycerol molecule. As a result, a very thin and low-shear-strength tribofilm is formed on the ta-C sliding surface. ${ }^{15,17,22}$ It appears that superlubricity is directly related to the trialcohol chemical functions ( $-\mathrm{OH})$ of the glycerol molecule. However, as shown in Fig. 2, in the case of ta-C at $80^{\circ} \mathrm{C}$, the addition of myo-inositol does not significantly improve the friction level.

\section{B. Mechanism of superlubricity of ta-C by glycerol by using molecular dynamics simulations}

After sliding the surface in the presence of glycerol, we found that the bare surface reacts with glycerol to form surface $\mathrm{OH}$ and surface OR bonds (where R contains $1-3 \mathrm{O}$ or $\mathrm{C})$. Second, we prepare a saturated $\mathrm{OH}$-terminated surface by allowing $\mathrm{H}_{2} \mathrm{O}_{2}$ molecules to react with ta-C. Then, we carried out sliding simulations by moving the top surface with respect to the bottom one. ${ }^{23}$

For the bare ta-C surface, we found friction coefficients up to 1.0 and tremendous wear. With $\mathrm{OH}$-terminated ta-C surface, the friction coefficient was reduced to $0.01-0.03$. Thus, hydroxylation on the surface dramatically decreases the friction, leading to superlow friction. We also study the reaction of glycerol molecules with $\mathrm{OH}$-terminated ta-C surface by adding four glycerol monolayers, each has six glyc-

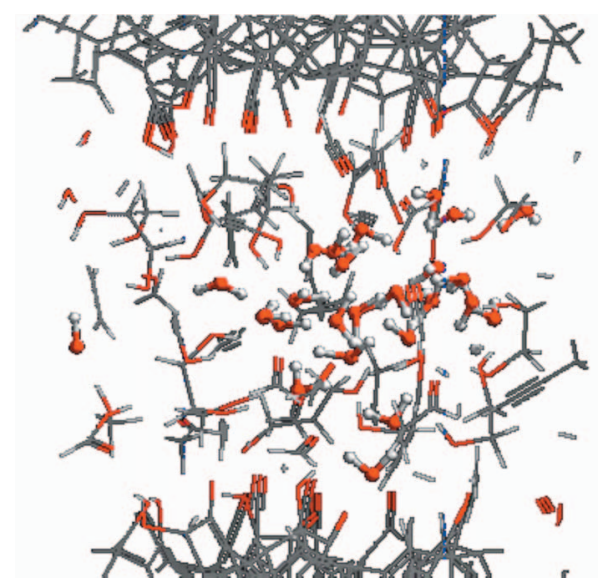

FIG. 3. (Color) Formation of water molecules by molecular dynamics simulation (shown with small circles) during lubrication of $\mathrm{OH}$-terminated ta-C/ta-C in presence of initially layered glycerol molecules. Contact pressure is $0.5 \mathrm{GPa}$ and surfaces were slid for 4 ps with a sliding speed of $1 \mathrm{ps} / \mathrm{nm}$

erol molecules, between the two ta- $\mathrm{C}$ slabs. When sliding the $\mathrm{OH}$-terminated ta-C friction couples in the presence of glycerol, we were surprised to observe the decomposition of glycerol and the formation of water and glycoaldehyde molecules in the interface, as shown in Fig. 3. The number of water molecules increases from 0 to about 14 after sliding 4 ps. The water molecules, formed by decomposition of glycerol, connect with $\mathrm{OH}$ groups on the surface by hydrogen bond, maybe sustaining the low friction of the surfaces.

\section{Mechanism of superlubricity of ta-C by glycerol using glycerol gas phase lubrication}

In this experiment, we used the ECT tribometer and the pressure of glycerol in the chamber was adjusted to $1 \mathrm{mbar}$ $(1 \mathrm{hPa})$. The test was performed with ta-C coated surfaces (pin and flat) at ambient temperature $\left(25^{\circ}\right)$ using the conditions described in the experimental part. For this particular experiment, we used deuterated glycerol of chemical formula $\mathrm{C}_{3} \mathrm{H}_{5}(\mathrm{OD})_{3}$. To measure the partial pressure of gas during the friction test, we used a derivation of the main chamber and the pressure was adjusted at $10^{-6} \mathrm{mbar}$ in order to use the residual gas analyzer (RGA). This is because the RGA cannot work at higher pressures. Mass spectra were recorded every $30 \mathrm{~s}$ during the friction test and we focused on peaks at mass $18\left(\mathrm{H}_{2} \mathrm{O}\right.$ and/or $\left.\mathrm{OD}\right)$ and at mass $20\left(\mathrm{D}_{2} \mathrm{O}\right)$. Figure 4 shows the evolution of the intensity of mass peaks during the duration of the test. It is clear that the peak corresponding to heavy water (at mass 20) increases while the peak attributed to water (or DO) at mass 18 decreases. This strongly suggests that heavy water is formed from the degradation of glycerol under the friction process.

At the end of the test, time-of-flight secondary ion mass spectroscopy (ToF-SIMS) analyses were performed inside the wear track and outside the wear track (as reference), respectively. Table I presents the intensities of several peaks corresponding to $\mathrm{H},{ }^{2} \mathrm{H}, \mathrm{OH}$, and $\mathrm{O}^{2} \mathrm{H}$. The results show a significant increase in the intensities of peaks associated with 


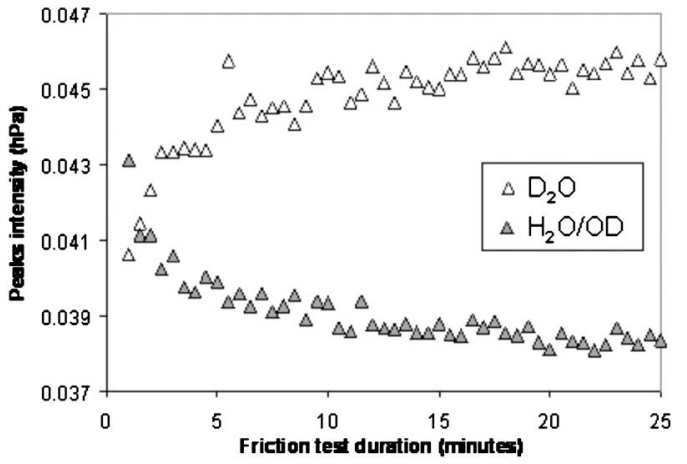

FIG. 4. Evolution of peak intensities at mass $18\left(\mathrm{H}_{2} \mathrm{O}\right.$ and/or OD) and at mass $20\left(\mathrm{D}_{2} \mathrm{O}\right)$ during a friction test performed with gaseous deuterated glycerol $\mathrm{C}_{3} \mathrm{H}_{5}(\mathrm{OD})_{3}$. The peak due to heavy water increases while peak due to water or DO decreases. This result strongly suggests the formation of water originating from the degradation of deuterated glycerol during friction test.

mass of ${ }^{2} \mathrm{H}$ and $\mathrm{O}^{2} \mathrm{H}$ peaks. This clearly confirms the hydroxylation/hydrogenation mechanism of the carbon surface under the friction process.

\section{Superlubricity of steel in presence of glycerol and myo- inositol}

Unfortunately, glycerol alone is found not to be able to durably lubricate the uncoated steel/steel friction pairs in the whole contact pressure range. Figure 5 shows friction curves of polished steel pairs lubricated by glycerol at different contact pressures and at ambient temperature $\left(25^{\circ} \mathrm{C}\right)$. It is interesting to notice that the highest contact pressure (about 1 $\mathrm{GPa}$ ) gives the better friction reduction. However, the low friction regime, which starts immediately, does not last for a long time and friction generally increases up to 0.1 after a few hundreds of cycles. This suggests a tribochemical reaction between the iron oxide layer and the alcohol. By accident, we stopped an experiment after 100 cycles without releasing the load and keeping the static contact conditions for about $2 \mathrm{~h}$. After this test, we were surprised to observe a mark in the contact area (corresponding to the Hertzian diameter) by optical microscopy (see Fig. 6). Observing this mark in the SEM shows a typical corrosion pattern on the surface with revelation of the chromium carbide grains in the

TABLE I. ToF-SIMS analyses in the wear track after a friction experiment with gaseous deuterated glycerol. A significant increase in the intensities of ${ }^{2} \mathrm{H}$ and $\mathrm{O}^{2} \mathrm{H}$ peaks is observed (compared to intensities of spectrum recorded outside the wear track). This strongly supports the hydroxylation and/or hydrogenation mechanism of ta-C surface during the friction process.

\begin{tabular}{|c|c|c|c|c|}
\hline & $\mathrm{H}$ & ${ }^{2} \mathrm{H}$ & $\mathrm{OH}$ & $\mathrm{O}^{2} \mathrm{H}$ \\
\hline $\begin{array}{l}\text { Outside } \\
\text { the wear } \\
\text { track }\end{array}$ & 3012841 & 10575 & 706662 & 1121 \\
\hline $\begin{array}{l}\text { Inside the } \\
\text { wear track }\end{array}$ & 3364705 & 105857 & 740762 & 3778 \\
\hline
\end{tabular}

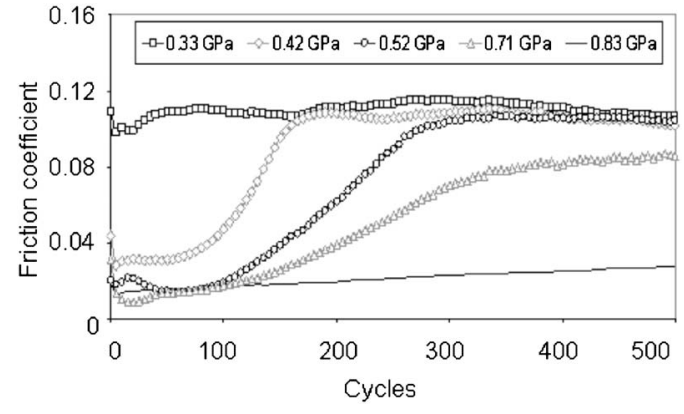

FIG. 5. Evolution of friction coefficient vs the number of cycles for highly polished steel/steel friction pairs lubricated with glycerol at ambient temperature and for different contact pressures. The friction tests were performed with the pin-on-flat tribometer at a sliding speed of $3 \mathrm{~mm} \mathrm{~s}^{-1}$ and with a track length of $2 \mathrm{~mm}$. The friction reduction mechanism is pressure dependent for glycerol lubricated steel systems. A high contact pressure is necessary to activate the tribochemical reaction.

steel structure. The presence of chromium in the grains was clearly evidenced by x-ray emission spectroscopy analysis in the SEM (not shown here). It is no question that this corrosion has been made by an acid or an aldehyde and not by glycerol because there is no evidence of corrosion of steel surface outside the contact zone, where the lubricant was also present during a long period. We can conclude that glycerol was present inside the contact area and has certainly been tribochemically transformed into some organic acids compounds. For example, three acids may be formed starting from the glycerol molecule: propionic acid $\mathrm{C}_{3} \mathrm{H}_{5} \mathrm{COOH}$, acetic acid $\mathrm{CH}_{3} \mathrm{COOH}$, and formic acid $\mathrm{HCOOH}$. The proposed chemical reactions are as follows:
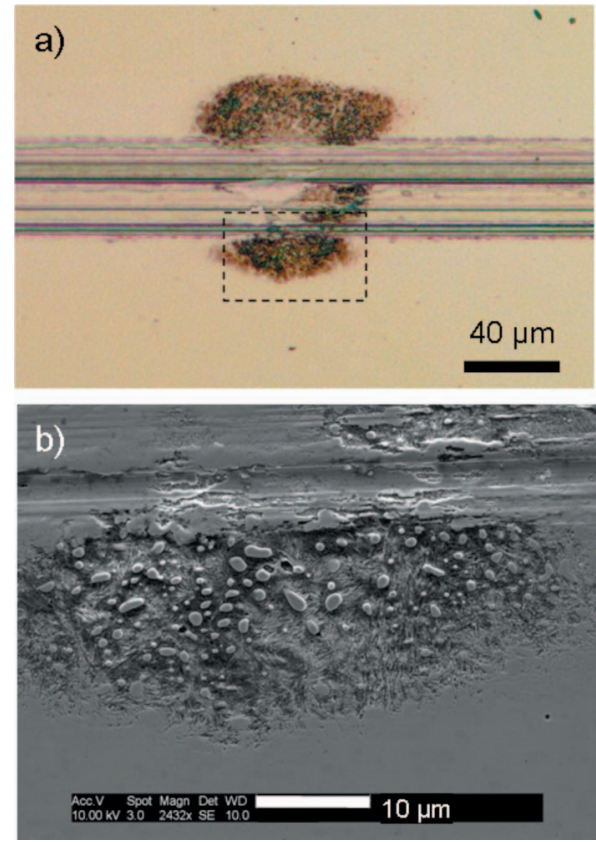

FIG. 6. (Color) Wear track observed on the flat after a friction test of 100 cycles with pure glycerol. The contact was kept closed without releasing the load during $2 \mathrm{~h}$. A mark was observed on the flat where the ball stayed static during the $2 \mathrm{~h}$. (a) Optical image, (b) SEM image of the dashed area. 


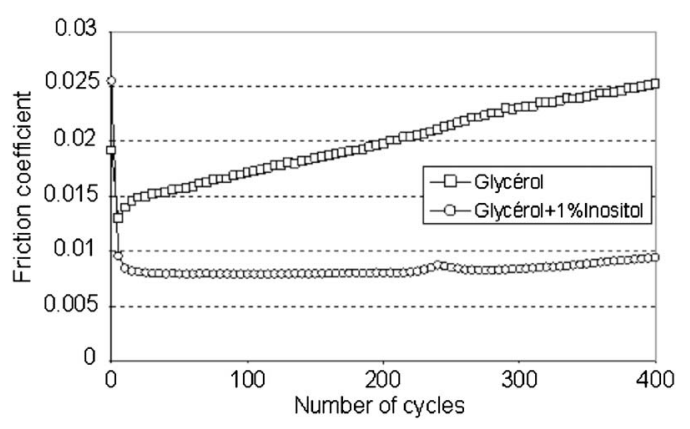

FIG. 7. Superlubricity of steel/steel friction pair lubricated by a drop of a solution of 1 mass \% myo-inositol in glycerol (at ambient temperature; $25^{\circ} \mathrm{C}$ ). The friction tests were performed with the reciprocating pin-on-flat tribometer with a sliding speed of $3 \mathrm{~mm} \mathrm{~s}^{-1}$, a track length of $2 \mathrm{~mm}$, and under a constant normal load of $10 \mathrm{~N}$ (corresponding to a maximum contact pressure of 800 $\mathrm{MPa}$ ). In these conditions of high pressure and considering surfaces roughness of $30 \mathrm{~nm}$, a glycerol viscosity of $934 \mathrm{mPa}$ and a glycerol pressure viscosity coefficient of $5.4 \mathrm{GPa}^{-1}$. The calculated minimum film thickness is about $10 \mathrm{~nm}$ and the lambda ratio is inferior to unity. So the lubrication regime is boundary and EHL lubrication is excluded. After 400 cycles, friction tends to increase because inositol has been consumed by the tribochemical reaction. Heating at $80{ }^{\circ} \mathrm{C}$ suppresses the superlow regime, probably by desorption of inositol molecules on the $\mathrm{OH}$-terminated surface.

$$
\begin{gathered}
\mathrm{C}_{3} \mathrm{H}_{5}(\mathrm{OH})_{3} \rightarrow \mathrm{C}_{2} \mathrm{H}_{5} \mathrm{COOH}+\mathrm{H}_{2} \mathrm{O}, \\
2 \mathrm{C}_{3} \mathrm{H}_{5}(\mathrm{OH})_{3} \rightarrow 2 \mathrm{CH}_{3} \mathrm{COOH}+2 \mathrm{H}_{2} \mathrm{O}+\mathrm{C}_{2} \mathrm{H}_{4}, \\
\mathrm{C}_{3} \mathrm{H}_{5}(\mathrm{OH})_{3} \rightarrow \mathrm{HCOOH}+\mathrm{H}_{2} \mathrm{O}+\mathrm{C}_{2} \mathrm{H}_{4} .
\end{gathered}
$$

Other acids such as glycolic acid and oxalic acid may also be formed. Other reactions can be found in the literature about the conversion of glycerol into glycerol acetate, carbon dioxide, and hydrogen, however, no corrosion is expected in this case. In our reactions, water molecules are formed and ethylene can also be released. When the acids formed leave the contact area after sliding, they come in contact with glycerol and can react to form glycerol acetate and water. Thus, corrosion of steel may not have the time to take place. This is corroborated by the fact that no corrosion was observed in the case of a static loaded contact (without sliding before). This observation strongly suggests that the degradation of glycerol under the combined effect of pressure and shear and water molecules released by the reaction are in agreement with the prediction of our MD calculations.

In Fig. 7, we compare the friction properties of steel surfaces lubricated by glycerol and glycerol+ 1 wt \% of myoinositol using the pin-on-flat tribometer. At ambient temperature, it is remarkable to see the positive effect of inositol in reducing friction of steel/glycerol system in the superlow regime. No other lubricant is found to reach such a friction value with steel/steel tribological system except in hydrodynamic lubrication. Compared to polar molecules under boundary lubrication, friction is about ten times lower, showing outstanding tribology performance. What is more, absolutely no wear track on steel is visible by optical microscopy.
It is thought that the mechanism of friction reduction of steel by inositol is similar to the case of ta- $\mathrm{C}$ with glycerol and that inositol molecules can also produce water molecules inside the contact area. MD simulations are currently under consideration. Moreover, we observed that in the presence of the solution of inositol, friction increases after a certain time and tends to reach the value of pure glycerol. If we add another drop of solution in the contact, then friction decreases again in the superlow regime. This strongly suggests that inositol has been consumed by the friction process, possibly in forming water and quinone according to the reaction:

$$
\mathrm{C}_{6} \mathrm{H}_{6}(\mathrm{OH})_{6} \rightarrow 4 \mathrm{H}_{2} \mathrm{O}+\mathrm{C}_{6} \mathrm{H}_{4} \mathrm{O}_{2} \text {. }
$$

\section{E. Model of superlubricity by glycerol}

Since the pioneering work of Tabor in the 1950s, the mechanism of friction reduction under boundary lubrication has always been attributed to the effect of long chain polar (amphiphilic) molecules. In the so-called "self-assembled monolayer (SAM)" or "monolayer" model, the polar extremity of the molecule is strongly chemisorbed on the native oxide layer present on the steel surface while the paraffinic moiety extends outside the metal surface. The amphiphilic molecules must have at least ten carbons in their aliphatic chain in order to promote the formation of a crystal-like structure in the monolayer. Then, low friction is generally attributed to the easy sliding of methyl groups of SAMs over each other, as described by molecular dynamics simulations of Harrison ${ }^{24}$ for example. The drawback of this situation is that the monolayer has a limited durability and molecules generally desorb at elevated temperature. Another problem is that superlow friction values (below 0.01) are only attained at very low contact pressures (below $10 \mathrm{MPa}$ in the surface force apparatus, for example). At several hundred MPa contact pressures, or more in macroscale situations, friction never drops down below 0.04. In our experiments, the SAM model cannot be applied since glycerol molecule has not such a long aliphatic chain. Then, there is an opportunity for another lubrication mechanism here, which we discuss in the following, in the light of our findings. The new model, which we call "H-bond network" model, is based on three chronological steps that we further describe in the case of a carbon surface (ta-C).

(1) Hydroxylation of the solid friction surface by a tribochemical reaction of nascent surface with alcohols groups leading to an $\mathrm{OH} / \mathrm{H}$-terminated surface of ta-C. This reaction is enhanced by contact pressure and temperature. The hydroxylation of surfaces by the tribochemical reaction was evidenced by ToF-SIMS experiments performed using liquid deuterated glycerol molecules. ${ }^{3,15}$ By using gas phase lubrication in this work, we show here even the best confirmation for this reaction (see Table I). Further evidence for the $\mathrm{OH}$ terminated surface is given by observing the very good wettability of the wear scar by pure water, the contact angle drastically decreasing inside the worn area compared to the virgin area. ${ }^{25}$

(2) Formation of a hydrogen bond network tribofilm con- 


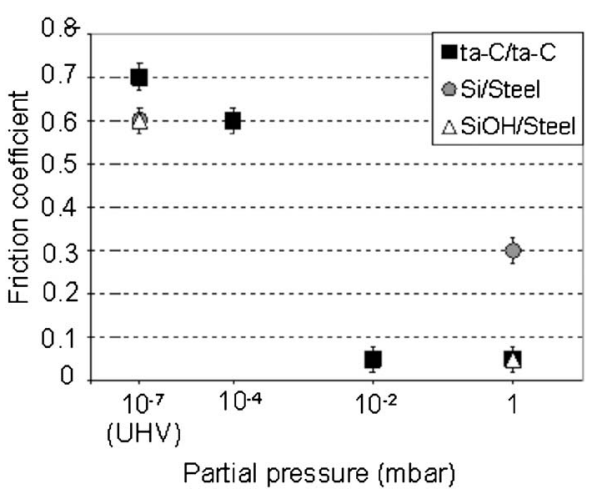

FIG. 8. Steady-state friction coefficient values for different friction pairs vs glycerol partial pressure in the ECT tribometer. All friction experiments were carried at $80{ }^{\circ} \mathrm{C}$. The sliding speed was 1 $\mathrm{mm} / \mathrm{s}$ and the track length was $2 \mathrm{~mm}$ under a normal load of $1 \mathrm{~N}$ (corresponding to a maximum contact pressure of $300 \mathrm{MPa}$ ). After the induction period corresponding to dangling bonds formation, the glycerol molecules react with the carbon surface providing efficient lubrication. This set of experiments also suggests that previously formed $\mathrm{OH}$-terminated surface $(\mathrm{Si}-\mathrm{OH})$ is important for the friction reduction of silicon by glycerol. Glycerol can hardly lubricate native and untreated silicon surfaces.

sisting of glycerol molecules adsorbed on the OH-terminated surface by $\mathrm{H}$ bond. This is more probable at a lower temperature. The presence of some residual glycerol molecules adsorbed on the $\mathrm{OH}$-terminated surface was first shown by ToF-SIMS analysis performed directly on the worn surface. The parent molecular ion $\mathrm{C}_{3} \mathrm{H}_{8} \mathrm{O}_{3}^{+}$, characteristic of the glycerol molecule is found in the ToF-SIMS mass spectrum, even after washing the surface. ${ }^{3,15}$ To give further evidence of the role of glycerol molecules on the OH-terminated surface, we performed friction tests using ta-C/taC, Si/steel, and Si-OH/ steel friction pairs at different gaseous partial pressures of glycerol in the ECT tribometer. For the silicon surface, the hydroxylation was obtained by plasma of water vapor. Friction data are summarized in Fig. 8. For virgin silicon surface, there is only a light lubrication effect by glycerol gas; at the opposite, friction of the $\mathrm{OH}$-terminated silicon surface was drastically reduced by glycerol molecules. This result strongly suggests that both $\mathrm{OH}$-terminated surface and the presence of glycerol molecules are important for the friction reduction mechanism.

(3) Tribodegradation of intercalated glycerol molecules, producing organic acids and water inside the contact. The acids formed can immediately react with glycerol at the exit of the contact to form glycerol acetate and water. However, acids can react with steel surface and cause corrosion. The main benefit effect on friction could be related to the existence of a lower viscosity interface film that is produced (compared to pure glycerol). This behavior was suggested by Raviv and Klein $^{26}$ who showed that bound water in hydration layers remain extremely fluid. The explanation of low friction by a "waterlike layer" itself is still speculative but has been proposed by Lee and Spencer, ${ }^{27}$ for example, in the case of polymer brushes. By using our high vacuum tribometer, we previously studied friction of steel on in situ fabricated amorphous ice as a function of temperature in the range of $100-0{ }^{\circ} \mathrm{C} .{ }^{28}$ We observed a clear reduction of friction when the temperature is above $-20^{\circ} \mathrm{C}$. It is well argued in the literature that, at this temperature, a liquid water molecular layer (typically 1-2 nm thick) is present on the solid ice surface. ${ }^{29}$ We attributed the friction reduction of this experiment to the sliding on the liquidlike water layer. In our MD experiment, we did not observe the general tendency to layering, a property of liquids confined between very smooth solid surfaces. ${ }^{30}$ At the opposite, the initially ordered glycerol layers were disordered, suggesting a more liquidlike response. Eventually, the worn surfaces were found hydrophilic. ${ }^{25}$ Moreover, in this case, it has been observed that water film confined between such hydrophilic surfaces are stable while water nanofilm is spontaneously ejected between two hydrophobic surfaces. ${ }^{31}$ In our case, we can only speculate on a synergistic effect of $\mathrm{OH}$-terminated surface with short chain polyol molecules eventually forming a low viscosity water-containing nanofilm in the hydrogen bond network system. What is particular in our experiments compared to other published works is that water molecules are generated inside the contact zone and they cannot be easily ejected. However, in our case, it is practically impossible to give full analytical proof for this phenomenon due to technological difficulties to detect very low quantities of water in glycerol. New experiment with deuterated glycerol and NMR spectroscopy are planed in the near future.

\section{CONCLUSIONS}

We present here a new tribological macrosystem that is able to reach superlubricity under practical conditions of boundary lubrication at ambient temperature. Typically, the use of steel surfaces and/or ta-C coated surfaces lubricated by selected polyhydric alcohols molecules can give unsurpassed low friction values below 0.01, approaching the socalled superlubricity regime (when sliding is approaching pure rolling). Previously, this performance had only been attained with $a-\mathrm{C}: \mathrm{H}$ and $\mathrm{MoS}_{2}$ coatings under ultrahigh vacuum or in inert gas atmosphere (dry friction regime). The mechanism of friction reduction is basically different from the one known for classical boundary lubrication by longchain polar molecules. The proposed new mechanism $(\mathrm{H}-$ bond network model) is based on the existence of a network of H-bond interactions in the confined interface zone.

First, the grafting of the friction surface by hydroxyl groups is necessary and we show that it is brought by a tribochemical reaction between the surface and the alcohol groups. This is well supported by ToF-SIMS analyses and computer simulations experiments.

Second, the polyols molecules adsorb on the $\mathrm{OH}-$ terminated surface, thanks to $\mathrm{H}$ bonds, and eventually intercalate between the two contacting $\mathrm{OH}$-terminated surfaces. The sliding occurs through rapid changes of H-bond locations in the whole network.

Third, we have strong experimental and numerical evidences of the tribodegradation of glycerol molecules under the combined effects of pressure and shear, generating aldehydes or organic acids and water molecules. At present, we 
can only speculate that superlow friction is related to the presence of a water-containing nanometer-thick tribofilm existing in the contact area, possibly by changing viscositypressure properties of the mixture. We observed that the tribodegradation of glycerol is detrimental in the steel on steel combination because of associated corrosive effects. In the case of ta-C coated surfaces, the formation of water could be an advantage.

There are many other polyhydric alcohols and cyclitols that would be certainly interesting in boundary lubrication and could be applied in macromachine, as well as medical devices.

\section{ACKNOWLEDGMENTS}

The authors would like to thank Nissan Motors Co. Ltd. for financial support for this research and Magali Phaner and Yann Chevolot from the LEOM Laboratory of Ecole Centrale de Lyon for providing hydroxylated and nonhydroxylated silicon wafer samples.
${ }^{1}$ M. Hirano and K. Shinjo, Phys. Rev. B 41, 11837 (1990).

${ }^{2}$ J. B. Sokoloff, Phys. Rev. B 42, 760 (1990).

${ }^{3}$ Superlubricity, edited by A. Erdemir and J. M. Martin (Elsevier, New York, 2007).

${ }^{4}$ F. P. Bowden and D. Tabor, The Friction and Lubrication of Solids (Clarendon, Oxford, 1964), Pts. 1 and 2, pp. 110-121 and 158-185.

${ }^{5}$ P. W. Bridgeman, Proc. Am. Acad. Arts Sci. 71, 387 (1936).

${ }^{6}$ I. Singer, R. N. Bolster, J. Wegang, S. Fayeulle, and B. C. Stupp, Appl. Phys. Lett. 57, 995 (1990).

${ }^{7}$ J. M. Martin, C. Donnet, T. Le Mogne, and T. Epicier, Phys. Rev. B 48, 10583 (1993).

${ }^{8}$ C. Donnet, T. Le Mogne, and J. M. Martin, Surf. Coat. Technol. 62, 406 (1993).

${ }^{9}$ T. Le-Mogne, C. Donnet, J. M. Martin, A. Tonck, N. MillardPinard, S. Fayeulle, and N. Moncoffre, J. Vac. Sci. Technol. A 12, 1998 (1994).

${ }^{10}$ J. M. Martin, H. Pascal, C. Donnet, T. Le-Mogne, J. L. Loubet, and T. Epicier, Surf. Coat. Technol. 68-69, 427 (1994).

${ }^{11}$ C. Donnet, J. M. Martin, T. Le-Mogne, and M. Belin, Tribol. Int. 29, 123 (1996).

${ }^{12}$ C. Donnet, J. Fontaine, T. Le-Mogne, M. Belin, C. Héau, J. P. Terrat, F. Vaux, and G. Pont, Surf. Coat. Technol. 120-121, 548 (1999).

${ }^{13}$ M. Chhowalla and G. A. J. Amaratunga, Nature (London) 407, 164 (2000).

${ }^{14}$ F. Ilie, Tribol. Int. 39, 774 (2006).

${ }^{15}$ M. I. de Barros Bouchet, C. Matta, T. Le Mogne, J. M. Martin, and T. Sagawa, Tribology - Materials, Surfaces and Interfaces 1, 28 (2007).

${ }^{16}$ J. Tannous, M. I. De Barros Bouchet, T. L. Mogne, P. Charles, and J. M. Martin, Tribology - Materials, Surfaces and Interfaces
1, 98 (2007).

${ }^{17}$ M. Kano, Y. Yasuda, Y. Okamoto, Y. Mabuchi, T. Hamada, T. Ueno, J. Ye, S. Konishi, S. Takeshima, J. M. Martin, M. I. De Barros Bouchet, and T. L. Mogne, Tribol. Lett. 18, 245 (2005).

${ }^{18}$ J. Robertson, Philos. Mag. B 76, 335 (1997).

${ }^{19}$ A. C. T. van Duin, S. Dasgupta, F. Lorant, and W. A. Goddard, J. Phys. Chem. A 105, 9396 (2001).

${ }^{20}$ A. C. T. van Duin, A. Strachan, S. Stewman, Q. S. Zhang, X. Xu, and W. A. Goddard III, J. Phys. Chem. A 107, 3803 (2003).

${ }^{21}$ C. Donnet, M. Belin, J. C. Augé, J. M. Martin, A. Grill, and V. Patel, Surf. Coat. Technol. 68-69, 626 (1994).

${ }^{22}$ Y. Yasuda, M. Kano, M. Mabuchi, and S. Abou, Proceedings of SAE, in Advanced Materials \& Processes, 2003, Paper No. 011101 .

${ }^{23}$ Q. Zhang, Y. Qi, L. G. Hector, T. Cagin, and W. A. Goddard III, Phys. Rev. B 72, 045406 (2005).

${ }^{24}$ J. A. Harrison, G. T. Gao, R. J. Harrison, G. M. Chateauneuf, and P. T. Mikulski, in Encyclopedia of Nanoscience and Nanotechnology, edited by H. S. Nalwa (American Scientific Publishers, Los Angeles, 2004), pp. 511-527.

${ }^{25}$ C. Matta, M. I. De Barros Bouchet, T. Le Mogne, B. Vacher, J. M. Martin, and T. Sagawa, Lubr. Sci. 20, 137 (2008).

${ }^{26}$ U. Raviv and J. Klein, Science 297, 1540 (2002).

${ }^{27}$ S. Lee and N. D. Spencer, Tribol. Int. 38, 922 (2005).

${ }^{28}$ H. Liang, J. M. Martin, and T. Le-Mogne, Acta Mater. 51, 2639 (2003).

${ }^{29}$ J. G. Dash, H. Y. Fu, and J. S. Wettlaufer, Rep. Prog. Phys. 58, 115 (1995).

${ }^{30}$ J. Gao, W. D. Luedtke, and U. Landman, Phys. Rev. Lett. 79, 705 (1997).

${ }^{31}$ J. N. Israelachvili, Intermolecular and Surface Forces, 2nd ed. (Academic, New York, 1991). 